\title{
Avaliação da qualidade dos registros de cartões de pré-natal de mulheres urbanas
}

\author{
Quality assessment of antenatal care home-based records of urban women \\ Evaluación de la calidad de los registros de tarjeta prenatal de mujeres urbanas
}

\section{Letícia Ferreira de Camargos $^{1}$ (i) Patrícia Lage Lemos ${ }^{1}$ (D) \\ Eunice Francisca Martins ${ }^{1}$ (D)}

Mariana Santos Felisbino-Mendes ${ }^{1}$ (1)

1. Universidade Federal de Minas Gerais, Escola de Enfermagem, Departamento de Enfermagem Materno-Infantil e Saúde Pública. Belo Horizonte, MG, Brasil.
Autor correspondente:

Mariana S Felisbino-Mendes.

E-mail: marianafelisbino@yahoo.com.br.

Recebido em 25/05/2020.

Aprovado em 07/08/2020.

DOI:https://doi.org/10.1590/2177-9465-EAN-2020-0166

\section{Resumo}

Objetivo: Avaliar registros dos cartões de pré-natal de puérperas atendidas em maternidades públicas e privadas. Método: Estudo transversal que analisou fotos de 394 cartões de pré-natal de mulheres que participaram da pesquisa Nascer em Belo Horizonte. Os registros foram avaliados quanto ao preenchimento, legibilidade e completude das informações sociodemográficas, clínicas, obstétricas e exames laboratoriais. Resultados: Evidenciou-se que 88,5\% dos cartões não apresentaram registro do nome da unidade básica de referência, 76,9\%, da maternidade de referência e $82,4 \%$, da instituição de realização do pré-natal A avaliação do edema, o uso de sulfato ferroso e ácido fólico não foram registrados em $55 \%, 91,1 \%$ e $92,6 \%$ dos cartões respectivamente. A ausência de registros no gráfico de peso foi identificada em $86,8 \%$ dos cartões, e no de altura uterina, $79,7 \%$. Conclusão e implicações para a prática: Existem grandes lacunas no registro do processo de cuidado pré-natal nos cartões e cadernetas, o que pode comprometer a qualidade da assistência e o acompanhamento das gestantes. Os achados apontam para a necessidade da educação permanente em serviço direcionada aos profissionais de saúde, como os enfermeiros, quanto à importância da realização e registro das ações preconizadas no cuidado pré-natal.

Palavras-chave: Cuidado Pré-Natal; Registros; Atenção Primária à Saúde; Enfermeiras e Enfermeiros; Saúde da Mulher.

\section{Abstract}

Objective: To evaluate the antenatal care (ANC) home-based records of puerperal women attended in public and private maternity hospitals. Methods: Cross-sectional study that analyzed photographs of 394 ANC home-based records of women who participated in the research Nascer em Belo Horizonte (Born in Belo Horizonte, in free translation). Records were assessed regarding completeness, legibility and completeness of sociodemographic, clinical, obstetric and laboratory data. Results: $88.5 \%$ of the cards had no record of the name of the primary care unit of reference, $76.9 \%$ of the maternity of reference and $82.4 \%$ of the ANC institution. Evaluation of edema, use of ferrous sulfate and folic acid were not registered in $55 \%, 91.1 \%$ and $92.6 \%$ of the cards, respectively. The absence of records in the weight chart was identified in $86.8 \%$ of the cards, and in the uterine height chart, $79.7 \%$. Conclusions and implications for practice: There are large gaps in the registration of the ANC process in the home-based records, which may compromise the quality of care and follow-up of pregnant women. The findings point to the need for permanent education in service directed to health professionals, such as nurses, regarding the importance of carrying out and registering the actions recommended in ANC

Keywords: Antenatal Care; Records; Primary Health Care; Nurses; Women's Health.

\section{Resumen}

Objetivo: Analizar registros de las tarjetas de prenatal de puérperas atendidas en maternidades públicas y privadas. Métodos Estudio transversal, con fotos de 394 tarjetas de prenatal, provenientes de la investigación Nascer em Belo Horizonte (Nacer en Belo Horizonte), que analizó registros en cuanto al llenado, legibilidad y completitud de las informaciones sociodemográficas, clínicas, obstétricas y exámenes de laboratorio. Resultados: El 88,5\% de las tarjetas no tuvieron registro del nombre de la unidad básica de referencia, el 76,9\%, de la maternidad de referencia y el 82,4\%, de la institución de realización del prenatal. Evaluación del edema, uso de sulfato ferroso y ácido fólico no se registraron en el $55 \%, 91,1 \%$ y $92,6 \%$ de las tarjetas, respectivamente. La ausencia de registros en el gráfico de peso fue identificada en el $86,8 \%$ de las tarjetas, y en el $79,7 \%$ de altura uterina. Conclusión: Existen grandes lagunas en el registro del proceso de asistencia prenatal, pudiendo comprometer la calidad de la asistencia y el acompañamiento de las gestantes. Implicaciones para la práctica: Se necesita educación permanente dirigida a profesionales de la salud, como enfermeras, sobre la importancia de llevar a cabo y registrar las acciones recomendadas en la atención prenatal.

Palabras clave: Atención Prenatal; Registros; Atención Primaria de Salud; Enfermeras y Enfermeros; Salud de la Mujer. 


\section{INTRODUÇÃO}

A assistência pré-natal constitui-se em um conjunto de ações destinadas às mulheres durante os períodos gestacional e puerperal, e ao concepto, contribuindo para a identificação de fatores que podem comprometer o bom desenvolvimento da gestação e, consequentemente, reduzir as taxas de morbimortalidade materna e fetal..$^{1,2}$ Para o acompanhamento da gestação e do seu desfecho, o cartão ou caderneta da gestante é um instrumento indispensável, pois contém o registro da condição de saúde materno-fetal e das ações realizadas durante as consultas. . $^{3,4}$

O Cartão da Gestante foi criado, no Brasil, em 1988, com o propósito de armazenar informações, facilitando o diálogo entre os profissionais que atuavam na assistência pré-natal e os que realizavam o parto nas maternidades. ${ }^{5}$ Seu uso se popularizou nos serviços de saúde pública, funcionando como um instrumento de interlocução entre os níveis de atenção. ${ }^{5}$

Atualmente, o registro na caderneta contribui também para uma comunicação efetiva entre os profissionais, tendo em vista a continuidade da assistência, especialmente quando há consultas de pré-natal intercaladas entre enfermeiros, médicos e especialistas, conforme preconizado na Estratégia Saúde da Família (ESF). ${ }^{6} \mathrm{O}$ uso desse registro também garante o direito de acesso à informação pelos usuários e está alinhado ao esforço global do cuidado centrado na pessoa. ${ }^{3}$ Ademais, durante a realização do cuidado pré-natal, as informações registradas na caderneta podem fundamentar a tomada de decisões durante a gestação, parto, pós-parto, e até mesmo nas próximas gestações. ${ }^{7}$ As informações permitem ainda uma avaliação longitudinal da gestação e sua evolução quanto aos parâmetros clínicos, como altura uterina, ganho de peso, pressão arterial, edema, dentre outros.

O cartão da gestante - no momento denominado caderneta - deve ser fornecido na primeira consulta de pré-natal, e preenchido a cada consulta de forma adequada, legível e completa, para cumprir o seu objetivo de meio de comunicação entre a gestante, os profissionais da rede básica de saúde e dos serviços de atendimento ao parto - ou do serviço de referência e contrarreferência, nos casos necessários. ${ }^{5}$ Outra relevância da caderneta da gestante é ser fonte de informação para o Sistema de Informações sobre Nascidos Vivos (SINASC). Tais informações são utilizadas em estudos que' avaliam a qualidade da assistência pré-natal e fundamentam políticas de saúde materna e infantil do Sistema Único de Saúde. ${ }^{8}$

Os registros das cadernetas são padronizados e englobam os antecedentes obstétricos, pessoais e familiares da mulher. Encerram, ainda, os exames preconizados para o primeiro, segundo e terceiro trimestres e as avaliações clínicas a cada consulta, incluindo os gráficos de altura uterina e ganho de peso. ${ }^{9}$ Portanto, a caderneta da gestante constitui, resumidamente, as recomendações pelos protocolos clínicos. Dessa forma, a ausência de registros nos cartões poderia expressar, indiretamente, a baixa qualidade da assistência pré-natal prestada, além de limitar as informações que contribuiriam na produção de indicadores que subsidiam melhor organização e planejamento da assistência ao pré-natal, e para o entendimento e alerta dos usuários acerca de sua saúde.

Nas últimas décadas, os serviços de atenção básica no Brasil vêm expandido e, atualmente, a cobertura da atenção pré-natal tem sido quase universal. ${ }^{10,11}$ Mais de $70,0 \%$ das gestantes iniciaram o pré-natal antes da 16. - semana de gestação, realizaram mais de seis consultas, e 96,0\% receberam a caderneta da gestante. ${ }^{10}$ Contudo, ainda persiste o desafio de melhorar a qualidade dessa assistência, mediante a oferta de todas as ações efetivas. ${ }^{12}$ Estudo prévio nacional demonstrou que cerca de $72,1 \%$ das gestantes chegaram às maternidades de posse desse documento. ${ }^{10}$ Ademais, os registros no cartão da gestante requerem melhorias, conforme sinalizado pelos poucos estudos sobre o tema, ${ }^{2,4,5}$ visto que a sua incompletude aponta inicialmente para a não realização da ação ou cuidado.

Em Belo Horizonte, a assistência pré-natal é ofertada em toda rede de atenção básica do Sistema Único de Saúde (SUS) e, como no restante do país, o acesso a esse serviço é universal. ${ }^{11}$ Considerando a importância do cartão, o seu uso como instrumento de apoio e registro do cuidado pré-natal, além de sua função essencial para a comunicação entre profissionais na rede de atenção e informação da mulher, reconhece-se a relevância de avaliar a situação do cartão da gestante quanto à qualidade do seu preenchimento. Estudos prévios propuseram avaliação semelhante. ${ }^{2,4,5,13,14}$ No entanto, as evidências acerca desse tema são ainda limitadas, pois analisam os dados da caderneta parcialmente ${ }^{2,4} \mathrm{e}$, na maioria das vezes, sintetizam a avaliação do preenchimento,,$^{2,4,13}$ o que pode mascarar graves faltas de preenchimento, além do dispêndio desse tipo de estudo. Outros analisaram documentos semelhantes, como o cartão da criança, ${ }^{15}$ mas até o momento as evidências são esparsas inclusive para sustentar fortemente o seu uso, apesar de já ter sido demonstrado, em recomendação internacional, que seus vários benefícios superam os danos. ${ }^{3}$

Assim, propõe-se, neste estudo, avaliar os registros nos cartões de pré-natal de puérperas atendidas em maternidades públicas e privadas de Belo Horizonte, quanto ao preenchimento, legibilidade e completude. Os resultados desta análise poderão contribuir para avanços na qualidade da caderneta da gestante, servir de parâmetro indireto de avaliação do cuidado pré-natal e fomentar a discussão sobre o tema registros na saúde. Ademais, este tema requer apontamentos contínuos, diante de sua importância técnica, ética e legal.

\section{MÉTODO}

Trata-se de estudo transversal, realizado a partir da amostra fotográfica de 394 cartões de pré-natal referentes a puérperas. Tais dados são provenientes da coorte de base hospitalar da pesquisa Nascer em Belo Horizonte: inquérito sobre parto e nascimento, realizada no período de novembro de 2011 a março de 2013, em 11 maternidades públicas e privadas do município de Belo Horizonte. Foram incluídas, no estudo, 1.088 puérperas que tiveram parto hospitalar de recém-nascido vivo, de qualquer peso ou idade gestacional, ou de natimorto, com peso superior a $500 \mathrm{~g}$ ou idade gestacional maior que 22 semanas. $O$ estudo avaliou a estrutura e processo das maternidades, as condições sociodemográficas, de saúde e de atenção ao pré-natal e parto das mulheres, bem como as condições ao nascer e assistência do recém-nascido na maternidade.

A coleta de dados foi realizada por meio de três instrumentos: um aplicado ao diretor da maternidade, outro às puérperas, e o terceiro para a busca dos dados no prontuário das mulheres e seus recém-nascidos, como também no cartão de pré-natal. As entrevistas com as puérperas ocorreram pelo menos seis horas após o parto, à beira leito, e a busca nos prontuários, após a alta da mulher e do recém-nascido. Os cartões de pré- 
natal foram fotografados após a entrevista com as puérperas, constituindo-se na fonte de dados para este estudo específico.

Os cartões foram considerados todo e qualquer documento que a puérpera apresentasse no momento da internação - seja do SUS ou do setor privado -, e que fornecessem informações sobre procedimentos e exames realizados durante o pré-natal, considerando os diferentes formatos, tamanhos e condições de conservação. As mulheres que tiveram suas cadernetas de pré-natal fotografadas foram 829. Porém, houve perdas devido ao não acesso à caderneta e a problemas técnicos na transferência dos dados - por exemplo, a qualidade da foto -, resultando na amostra de conveniência de 394 cadernetas, de puérperas de cinco maternidades. Após análise de sensibilidade - comparando as características gerais entre as amostras -, observou-se que a população estudada manteve características similares à amostra geral.

Para sistematizar a avaliação do preenchimento, legibilidade e completude dos cartões, optou-se pela construção de uma base de dados, a partir de um instrumento contendo 64 variáveis, elaborado em formato de planilha. Essas variáveis se basearam nos itens que compõem a caderneta da gestante e referem-se à identificação e procedência da mulher, história obstétrica prévia e atual, história clínica pregressa, história clínica da atual gestação, e o uso da suplementação de Ácido Fólico e Sulfato Ferroso. Também foi investigada a quantidade e a qualidade dos registros da avaliação clínica da gestante em cada consulta, incluindo pressão arterial, edema, e os gráficos de Altura Uterina (AU) e Índice de Massa Corporal (IMC), segundo Idade Gestacional (IG) e exames laboratoriais do primeiro, segundo e terceiro trimestres.

As fotos foram avaliadas individualmente, em média eram $5 \mathrm{a} 6$ fotos por cartão de pré-natal, totalizando aproximadamente 2.400 fotos. A planilha foi preenchida com códigos padronizados, conforme a seguir: 0 - Variáveis quantitativas em branco; 1 - Registro completo ou registrado; 2 - Registro incompleto; 3 - Registro ilegível; 4 - Sem registro; 5 - Não se aplica; 6 - Registrado em outro trimestre; 7-Problemas com a foto; 8 - Exames incompletos e registrados em outro trimestre. A técnica de avaliação das fotos dos cartões para extração dos dados foi padronizada, exercida por duas avaliadoras e, ao final do procedimento, realizou-se uma conferência cruzada de $40 \%$ de todos os cartões para revisão dos dados. A extração dos dados relativos ao preenchimento do cartão ocorreu nos meses de janeiro a junho de 2017.

Os dados foram analisados por meio do cálculo de frequências absolutas e relativas. Realizaram-se as análises com o auxílio do programa estatístico STATA versão 14.0 (Stata Corp., College Station, TX, USA).

O estudo Nascer em Belo Horizonte foi aprovado pelo Comitê de Ética em Pesquisa, da Universidade Federal de Minas Gerais (UFMG), sob o Parecer CAAE- 0246.0.203.000-11. Antes da entrevista, da coleta de dados do prontuário e fotografias das suas cadernetas de pré-natal, as mulheres assinaram Termo de Consentimento Livre e Esclarecido (TCLE); e referente às adolescentes, o responsável legal assinou o Termo de Assentimento Livre e Esclarecido (TALE).

\section{RESULTADOS}

Do total de 394 participantes do presente estudo, $62,2 \%$ das mulheres declararam ser de Belo Horizonte e 37,8\% de outras cidades, a maioria da região metropolitana. A média da idade \pm desvio-padrão das mulheres participantes foi de 27 anos $\pm 6,5$ anos, sendo a mínima 15 anos, e a máxima, 46 anos. Cerca de 10,7\% das mulheres tinha idade entre 14-24 anos, $47,2 \%$ entre $25-34$ anos e $41,6 \% 35$ anos ou mais. Quanto à cor da pele, a maioria se declarou parda, morena ou mulata (64\%). Sobre o grau de escolaridade, $85 \%$ tinham até o ensino médio completo. A maioria das mulheres realizou o pré-natal com o profissional médico (82,2\%), em instituições públicas $(68,3 \%)$, e 6 ou mais consultas de pré-natal (86,5\%). O parto ocorreu em hospitais públicos para $79,2 \%$ das puérperas, e a prevalência de cesariana foi de $58,9 \%$.

$\mathrm{Na}$ avaliação dos dados sociodemográficos e história prévia da gestante, as variáveis com maior número de ausência de registros foram: a Unidade Básica de Saúde (UBS) de referência (88,5\%); a maternidade de referência (76,9\%); a instituição de realização do pré-natal (82,4\%); e se a gravidez foi planejada ou não $(99,2 \%)$ (Tabela 1$)$.

Em relação aos registros de avaliação clínica em cada consulta, todos, à exceção do edema, obtiveram registros superiores a $97,7 \%$ (Tabela 2 ).

Na Tabela 3, observa-se que houve registro da realização de 6 ou mais consultas em 308 cartões (77,9\%). Os parâmetros clínicos pressão arterial, idade gestacional, peso e batimento cardíaco fetal foram registrados em 6 ou mais consultas, representando cerca de $75,0 \%$ dos casos. Observou-se que 280 cartões $(70,8 \%)$ apresentara o registro de altura uterina 6 vezes ou mais e, em 216 cartões $(54,8 \%)$, o parâmetro edema não foi registrado nenhuma vez.

Ao analisar os dados quantitativos (número de vezes) referentes ao preenchimento das curvas nos gráficos de peso/ idade gestacional (IMC/IG) e altura uterina/idade gestacional (AU/IG), observa-se ausência de registro em ambas as curvas. Ou seja, não foram preenchidas nenhuma vez em 95,2\% para IMC/ IG e em 94,9\% para AU/IG dos cartões (dados não mostrados). Apenas 4 cartões $(1,1 \%)$ tiveram mais de 6 registros para IMC/ IG e 2 para $A U / I G$.

Na análise dos registros dos exames laboratoriais nos cartões de pré-natal, observou-se baixa frequência de registros dos exames do primeiro, segundo e terceiro trimestres. Os exames do primeiro trimestre que obtiveram frequência igual ou maior que $50 \%$ de registros completos foram: urina rotina $(57,8 \%)$, glicemia jejum (61,9\%), HBsAg (57,6\%), Anti-HIV (57,6\%), VDRL $(60,4 \%)$, hemograma $(52,7 \%), A B O-R h(61,4 \%)$ e toxoplasmose $(58,8 \%)$ (Tabela 4$)$. Nenhum exame registrado no terceiro trimestre obteve frequência igual ou maior que $50 \%$ de registro completo.

Observaram-se registros de uso de sulfato ferroso suplementar em $6,9 \%$ dos cartões $(n=27)$. Já o uso de ácido fólico foi registrado em $5,1 \%$ ( $n=20)$ (dados não mostrados).

Em relação ao registro completo dos cartões, após análise do total de 394, apenas um apresentou registros de todas as variáveis e parâmetros clínico-obstétricos. 
Tabela 1. Avaliação da completude e legibilidade dos registros nos cartões de pré-natal: informações sociodemográficas, história obstétrica e história pregressa.

\begin{tabular}{lccccc}
\hline \multirow{2}{*}{$\begin{array}{c}\text { Informações da caderneta de } \\
\text { pré-natal }\end{array}$} & \multicolumn{5}{c}{ Registros no cartão de pré-natal } \\
\cline { 2 - 6 } & Completo n(\%) & $\begin{array}{c}\text { Incompleto } \\
\mathrm{n}(\%)\end{array}$ & Ausente $\mathrm{n}(\%)$ & llegível n(\%) & $\begin{array}{c}\text { Não se aplica } \\
\mathrm{n}(\%)\end{array}$ \\
\hline Idade da mulher & $355(90,1)$ & - & $36(9,1)$ & $03(0,7)$ & - \\
\hline Escolaridade & $234(59,3)$ & - & $155(39,3)$ & $05(1,2)$ & - \\
\hline Estado civil & $239(60,6)$ & - & $150(38,0)$ & $05(1,2)$ & - \\
\hline Unidade Básica de Referência & $44(11,1)$ & - & $349(88,5)$ & $01(0,2)$ & - \\
\hline Maternidade de referência & $88(22,8)$ & - & $303(76,9)$ & $03(0,7)$ & - \\
\hline Instituição de realização do PN & $68(17,2)$ & - & $325(82,4)$ & $01(0,2)$ & - \\
\hline Gestação prévia & $361(91,6)$ & - & $22(5,5)$ & $02(0,5)$ & $09(2,2)$ \\
\hline Parto vaginal prévio & $210(53,3)$ & $02(0,51)$ & $73(18,5)$ & $03(0,7)$ & $106(26,9)$ \\
\hline Cesariana prévia & $152(38,5)$ & $02(0,51)$ & $93(23,6)$ & $03(0,7)$ & $144(36,5)$ \\
\hline Abortos prévios & $221(56,0)$ & - & $90(22,8)$ & $03(0,7)$ & $80(20,3)$ \\
\hline Gravidez planejada ou não & $01(0,2)$ & - & $391(99,2)$ & $02(0,5)$ & - \\
\hline Doenças prévias & $271(68,7)$ & - & $121(30,7)$ & $02(0,5)$ & - \\
\hline Alergias & $33(8,3)$ & - & $360(91,3)$ & $01(0,2)$ & - \\
\hline
\end{tabular}

*PN: Pré-natal

Tabela 2. Avaliação da completude e legibilidade dos registros nos cartões de pré-natal: parâmetros da avaliação clínica.

\begin{tabular}{lcccc}
\hline \multicolumn{5}{c}{ Registros no cartão de pré-natal } \\
\hline \multicolumn{1}{c}{ Parâmetros clínicos } & Completo n(\%) & Incompleto n(\%) & Ausente n(\%) & Ilegível $n(\%)$ \\
\hline Idade gestacional & $388(98,4)$ & - & $03(0,7)$ & $03(0,7)$ \\
Peso & $388(98,4)$ & $01(0,2)$ & $03(0,7)$ & $02(0,5)$ \\
Altura uterina & $385(97,7)$ & $03(0,7)$ & $04(1,0)$ & $02(0,5)$ \\
Edema & $174(44,1)$ & $01(0,2)$ & $217(55,0)$ & $02(0,5)$ \\
Pressão arterial & $392(99,4)$ & - & - & $02(0,5)$ \\
Batimentos cardíacos fetais & $391(99,2)$ & - & - & $03(0,7)$ \\
\hline
\end{tabular}

Tabela 3. Número de consultas e número de vezes que os parâmetros clínicos foram registrados na caderneta da gestante durante o pré-natal.

\begin{tabular}{cccccccc}
\hline $\begin{array}{c}\mathrm{N} .{ }^{\circ} \text { de } \\
\text { vezes }\end{array}$ & $\begin{array}{c}\text { Consultas n } \\
(\%)\end{array}$ & $\begin{array}{c}\text { Avaliações de } \\
\text { IG n (\%) }\end{array}$ & $\begin{array}{c}\text { Avaliações de } \\
\text { Peso n (\%) }\end{array}$ & $\begin{array}{c}\text { Avaliações de } \\
\text { AU n (\%) }\end{array}$ & $\begin{array}{c}\text { Avaliações de } \\
\text { edema n (\%) }\end{array}$ & $\begin{array}{c}\text { Avaliações de } \\
\text { PA n (\%) }\end{array}$ & $\begin{array}{c}\text { Avaliações } \\
\text { de BCF n (\%) }\end{array}$ \\
\hline 0 & $01(0,2)$ & $05(1,2)$ & $05(1,2)$ & $06(1,5)$ & $216(54,8)$ & $01(0,2)$ & $01(0,2)$ \\
\hline 1 & $05(1,2)$ & $07(1,7)$ & $06(1,5)$ & $08(2,0)$ & $11(2,7)$ & $06(1,5)$ & $07(1,7)$ \\
\hline 2 & $06(1,5)$ & $05(1,2)$ & $07(1,7)$ & $10(2,5)$ & $14(3,5)$ & $09(2,2)$ & $07(1,7)$ \\
\hline 3 & $15(3,8)$ & $17(4,3)$ & $16(4,0)$ & $19(4,8)$ & $11(2,7)$ & $17(4,3)$ & $21(5,3)$ \\
\hline 4 & $24(6,0)$ & $24(6,0)$ & $28(7,1)$ & $37(9,3)$ & $12(3,0)$ & $28(7,1)$ & $29(7,3)$ \\
\hline 5 & $35(8,8)$ & $38(9,6)$ & $36(9,1)$ & $34(8,6)$ & $15(3,8)$ & $32(8,1)$ & $33(8,3)$ \\
\hline 6 & $44(11,1)$ & $49(12,4)$ & $53(13,4)$ & $61(15,4)$ & $28(7,1)$ & $50(12,6)$ & $52(13,2)$ \\
\hline ou + & $264(66,8)$ & $249(62,9)$ & $243(61,4)$ & $219(55,4)$ & $87(21,8)$ & $251(63,4)$ & $244(61,5)$ \\
\hline
\end{tabular}

*BCF: Batimentos cardiofetais; IG: Idade Gestacional; AU: Altura Uterina; PA: Pressão arterial 
Tabela 4. Completude dos registros dos exames laboratoriais no cartão de pré-natal.

\begin{tabular}{|c|c|c|c|c|c|}
\hline Exames laboratoriais & Completo n(\%) & Incompleto $\mathrm{n}(\%)$ & Ausente $n(\%)$ & $\begin{array}{c}\text { Registro } \\
\text { completo em } \\
\text { outro trimestre } \\
\mathrm{n}(\%)\end{array}$ & llegível n(\%) \\
\hline Urina rotina 1 & $228(57,8)$ & $12(3,0)$ & $80(20,3)$ & $63(15,9)$ & $11(2,8)$ \\
\hline Urocultura 1 & $170(43,1)$ & $07(1,7)$ & $155(39,3)$ & $52(13,2)$ & $10(2,5)$ \\
\hline Glicemia jejum 1 & $244(61,9)$ & $18(4,5)$ & $50(12,6)$ & $75(19,0)$ & $07(1,78)$ \\
\hline HBsAg 1 & $227(57,6)$ & $16(4,0)$ & $70(17,7)$ & $73(18,5)$ & $08(2,0)$ \\
\hline Anti Hiv 1 & $227(57,6)$ & $14(3,5)$ & $80(20,3)$ & $64(16,2)$ & $09(2,2)$ \\
\hline VDRL 1 & $238(60,4)$ & $12(3,0)$ & $57(14,4)$ & $79(20,0)$ & $08(2,0)$ \\
\hline Toxoplasmose 1 & $232(58,8)$ & $18(4,5)$ & $62(15,7)$ & $75(19,0)$ & $07(1,7)$ \\
\hline Hemograma completo 1 & $208(52,7)$ & $55(13,9)$ & $56(14,2)$ & $68(17,2)$ & $07(1,7)$ \\
\hline $\mathrm{ABO} / \mathrm{Rh} 1$ & $242(61,4)$ & $18(4,5)$ & $53(13,4)$ & $74(18,7)$ & $07(1,7)$ \\
\hline $\begin{array}{l}\text { Registro de todos os exames } \\
\text { do 1.o trimestre }\end{array}$ & $123(31,2)$ & $142(36,0)$ & $45(11,4)$ & $31(7,8)$ & $07(1,7)$ \\
\hline Teste de tolerância a glicose 2 & $111(28,1)$ & $03(0,7)$ & $68(17,2)$ & $18(4,5)$ & $12(3,8)$ \\
\hline Coombs indireto 2 & $32(8,1)$ & - & $57(14,4)$ & $03(0,7)$ & $09(2,2)$ \\
\hline Urina rotina 3 & $132(33,5)$ & $02(0,5)$ & $204(51,7)$ & $43(10,9)$ & $13(3,3)$ \\
\hline Urocultura 3 & $139(35,2)$ & $04(1,0)$ & $203(51,5)$ & $38(9,6)$ & $10(2,5)$ \\
\hline Glicemia jejum 3 & $145(36,8)$ & $03(0,7)$ & $195(49,4)$ & $42(10,6)$ & $9(2,2)$ \\
\hline HBsAg 3 & $84(21,3)$ & - & $274(69,5)$ & $25(6,3)$ & $11(2,8)$ \\
\hline Anti HIV 3 & $101(25,6)$ & $1(0,2)$ & $258(65,4)$ & $24(6,0)$ & $10(2,5)$ \\
\hline VDRL 3 & $179(45,4)$ & $1(0,2)$ & $166(42,1)$ & $38(9,6)$ & $10(2,5)$ \\
\hline Hemograma completo 3 & $150(38,0)$ & $23(5,8)$ & $169(42,8)$ & $41(10,4)$ & $11(2,8)$ \\
\hline Toxoplasmose 3 & $114(28,9)$ & $01(0,2)$ & $228(57,8)$ & $41(10,4)$ & $10(2,5)$ \\
\hline $\begin{array}{l}\text { Registro de todos os exames } \\
\text { do } 3 . \text { o trimestre }\end{array}$ & $17(4,3)$ & $210(53,3)$ & $123(31,2)$ & $10(2,5)$ & $10(2,5)$ \\
\hline
\end{tabular}

*Exames do primeiro (1), segundo (2) e terceiro (3) trimestres

\section{DISCUSSÃO}

Os resultados obtidos neste estudo evidenciaram qualidade insatisfatória da completude e legibilidade dos cartões de pré-natal das gestantes, e elevada a ausência de registros de identificação e ações executadas nas cadernetas. Em relação aos serviços de referência, é recomendado que a maternidade referenciada para o parto deve estar explícita no cartão da gestante, ${ }^{6}$ a fim de direcioná-la ao serviço de acordo com a complexidade demandada de forma individual. Contudo, a mulher tem a possibilidade de buscar atendimento em outro local. O referenciamento da gestante na rede evita o problema da peregrinação, ou seja, a necessidade de a gestante precisar passar por mais de uma unidade, em busca de atendimento para o parto, ${ }^{16}$ além de garantir o direito de acesso à informação e se alinhar ao cuidado centrado na pessoa - no caso, a mulher. ${ }^{3}$ Essa busca pela maternidade pode levar a desfechos desfavoráveis à mulher e ao feto, devido a inadequações no atendimento das suas necessidades. ${ }^{11,16}$

Por sua vez, o registro da Unidade Básica de Referência principalmente nos pré-natais acompanhados no Sistema Único de Saúde (SUS) - permite fazer referência e contrarreferência, e a alta responsável para continuidade dos cuidados no puerpério e ao recém-nascido. Uma hipótese que justificaria a desvalorização do registro dos dados relacionados à rede de atenção, é que ela pode não estar bem pactuada entre os níveis de assistência - especialmente para as gestantes não residentes em Belo Horizonte -, ou, ainda, que os profissionais desconhecem a rede de atenção pactuada.

O sub-registro da gravidez planejada ou não foi um achado negativo neste estudo, o qual pode impactar na forma de conduzir o cuidado pré-natal e nas taxas de morbimortalidade da mulher 
e criança. É fato que gravidez não planejada representa risco, aumentado de ansiedade e depressão, especialmente no período puerperal. ${ }^{12}$ Além disso, quando a gravidez não é planejada, é necessário que o profissional se empenhe mais em promover a vinculação dessa mulher ao acompanhamento pré-natal. Esse empenho reforça as orientações quanto à importância das ações do pré-natal e assiduidade às consultas, sendo fundamental, quando houver descontinuidade do acompanhamento, a realização de busca ativa. Desse modo, é indispensável que os profissionais entendam e registrem as circunstâncias em que a gestação está sendo desenvolvida. Ressalta-se inclusive que a caderneta pode facilitar o conhecimento da mulher em relação à sua própria condição de saúde, seus riscos, levando-a a se engajar mais adotando comportamentos saudáveis. ${ }^{3}$

Referente à avaliação clínica-obstétrica, no presente estudo, obtiveram-se dados de registros satisfatórios para alguns parâmetros em detrimento de outros. A altura uterina foi um parâmetro com alta frequência de registros, tendo prevalência similar aos dados de um estudo realizado em Juiz de Fora, Minas Gerais. ${ }^{17} \mathrm{~A}$ avaliação desse parâmetro é importante para identificar situações de restrição de crescimento intrauterino após a vigésima semana de gestação. Em contrapartida, a grande maioria dos cartões não teve registro da avaliação do edema, sinal clínico relevante que pode indicar risco gestacional, relacionado às síndromes hipertensivas e diabetes mellitus, dentre outras complicações..$^{18} \mathrm{O}$ registro insatisfatório do edema, encontrado neste estudo, também foi constatado em outro estudo realizado na região Sudeste. ${ }^{17}$ Ressalta-se que, dentre os cartões analisados, verificou-se maior ocorrência do preenchimento naqueles em que havia o campo para o registro do edema, se comparado aos que não tinham esse campo. Apesar de os campos direcionarem e influenciarem a avaliação do profissional, este deve ter ciência da importância de avaliar e registrar o surgimento do edema em todas as consultas.

A pressão arterial foi o parâmetro clínico mais registrado, em todas as consultas, na maioria dos cartões analisados. O batimento cardíaco fetal e o peso da gestante, em cada consulta, também obtiveram altas taxas de registros. Similarmente, em estudo realizado no município do Rio de Janeiro, os resultados apontaram registro elevado desses procedimentos, principalmente o da pressão arterial. Das gestantes, $96,3 \%$ confirmaram, por meio de entrevista, que tiveram a pressão arterial aferida. ${ }^{19} \mathrm{Essa}$ alta adesão em avaliar a pressão arterial possivelmente é em decorrência da hipertensão ser a complicação clínica mais comum na gestação, ocorrendo em 10 a $22 \%$ das gestantes, frequentemente associada a desfechos maternos e perinatais desfavoráveis. ${ }^{18,20}$

Sabe-se que a avaliação da pressão arterial é obrigatória; entretanto, deve ser associada a outros parâmetros clínicos, para indicar ou não alguma complicação na gestação. A exemplo, para avaliar o maior risco de pré-eclâmpsia, a pressão arterial deve ser avaliada juntamente com o ganho de peso súbito e/ ou a presença de edema. ${ }^{6,18}$ Ressalta-se a necessidade de os profissionais fazerem registros detalhados - no caso do edema-, descrevendo a sua localização e tipo, para que haja melhor diferenciação do edema fisiológico do patológico.

O peso foi um parâmetro que neste estudo também obteve altos índices de registros em todas as consultas. Tal resultado está em concordância com outro estudo realizado em Belo Horizonte. ${ }^{13}$ No entanto, o preenchimento da curva do ganho de peso gestacional foi precário, apesar de ter mais registros do que a curva da altura uterina segundo a idade gestacional. Esse baixo percentual de preenchimento das curvas foi também encontrado em estudo realizado no Rio Grande do Norte. ${ }^{14} \mathrm{O}$ preenchimento insuficiente das curvas dificulta o acompanhamento da evolução da gestação e a identificação de desvios no desenvolvimento fetal. ${ }^{14} \mathrm{Faz}$-se necessário que os profissionais de saúde realizem os procedimentos e, consequentemente, seus registros, na lógica da clínica; ou seja, de sua aplicabilidade. Dessa forma, fará sentido pesar, medir e registrar as curvas de ganho de peso para as tomadas de decisões.

Ao analisar o preenchimento dos exames laboratoriais, detectou-se sub-registro dos exames do primeiro, segundo e terceiro trimestres. Ressalta-se uma importante redução nos registros dos exames, do primeiro para o terceiro trimestre cerca de um terço -, corroborando com os achados de estudo transversal realizado em dois hospitais da rede pública de Belo Horizonte,,$^{13} \mathrm{e}$ com os resultados nacionais do inquérito Nascer no Brasil. ${ }^{10}$

Questiona-se, portanto, a razão pela qual os profissionais valorizaram e registram alguns parâmetros - como a pressão arterial e BCF-, em detrimento de outros, como o sub-registro do edema e da $\mathrm{AU}$, visto que todos são relevantes para a prática clínica e qualifica a atenção pré-natal. Cogitam-se algumas hipóteses, como a desvalorização das repercussões, em decorrência de complicações identificadas por meio desses parâmetros; ou o modelo de atenção, que confere maior valorização de parâmetros avaliados mediante o uso de tecnologia dura - como esfigmomanômetro e doppler. Nesse sentido, é importante reafirmar a essência do trabalho em saúde como trabalho vivo; ou seja, envolve a capacidade e a autonomia do profissional em transformar a tecnologia dura, instrumentos $\mathrm{e}$ equipamentos, e seus saberes em um modo próprio de fazer. Esse fazer se efetiva em ações e relações distintas, as quais são decorrentes, dentre outros fatores, da organização e da lógica predominante no processo de trabalho vigente, bem como do sentido atribuído aos seus produtos. ${ }^{21}$ Assim, torna-se valioso, no cuidado pré-natal, discutir o sentido e o valor da avaliação de cada parâmetro clínico e seu registro, a fim de atender às necessidades das mulheres e a vigilância dos riscos gestacionais.

Os achados do presente estudo apontam para a utilização insatisfatória da caderneta pelos profissionais de saúde lotados nos serviços de atenção à gestante, incluindo a Atenção Primária à Saúde. Estudo similar, que analisou o cartão da criança, também encontrou baixa frequência de preenchimento dos itens essenciais ao acompanhamento da criança. ${ }^{15}$ Análise de quatro inquéritos no Rio Grande do Sul mostrou preenchimento variável ao longo do tempo, apresentando quase universalização do 
preenchimento de alguns itens e outros muito aquém. ${ }^{4}$ Metanálise recente da Organização Mundial de Saúde enumerou vários motivos demonstrados como possíveis causas de sub-registro desses documentos, por parte dos profissionais, tais como estar muito ocupado, ou percebê-lo como um trabalho a mais. Incluem-se, ainda, não ter recebido treinamento adequado e achar que a mulher não lerá a caderneta, a esquecerá em casa quando for ao serviço para se consultar, ou até mesmo perderá o documento. ${ }^{3}$ Ressalta-se que o fato de a mulher não valorizar esse documento pode ser por desconhecimento de sua importância, devido à falta de orientação, ou pela desvalorização pelo próprio profissional.

A auditoria do Cartão ou Caderneta da Gestante tem sido utilizada para avaliar a qualidade dos cuidados oferecidos no prénatal e o cumprimento dos critérios previamente definidos. Nesse sentido, considera que a ausência do registro implica a inferência da não realização do procedimento ou exame complementar. ${ }^{22}$ No entanto, apesar de significativo valor para a avaliação do cuidado pré-natal, poucos estudos propuseram esse tipo de análise, possivelmente por ser dispendioso e demorado. A avaliação é importante, pois, apesar da elevada cobertura de pré-natal no Brasil - atingindo níveis praticamente universais -, a maioria das regiões apresenta assistência inadequada. ${ }^{23}$ Reforça-se, ainda, a recomendação recente da Organização Mundial de Saúde de utilizar esse tipo de registro e documento complementarmente aos registros que permanecem no serviço de saúde, tendo por base evidências - ainda que limitantes - dos efeitos desejáveis superarem os efeitos indesejáveis em relação ao seu uso na assistência materno-infantil. ${ }^{3}$

Acredita-se que esses problemas de preenchimento da caderneta da gestante compromete a integralidade da atenção, pois impede boa articulação da atuação entre os diversos profissionais envolvidos na assistência pré-natal, e afeta a Iongitudinalidade do cuidado dentro da rede de atenção à saúde. Essa continuidade do cuidado destaca-se no Brasil, que ainda apresenta elevada mortalidade materna, ligadas a causas passíveis de prevenção pela adequada atenção prénatal, como as doenças hipertensivas e infecções. ${ }^{11}$ Dessa forma, os registros da caderneta devem estar integrados ao sistema de informação em saúde e complementar os registros mantidos nos respectivos serviços, ${ }^{3}$ sendo meio de interlocução entre os profissionais da equipe de saúde. Os profissionais de enfermagem, como integrantes da equipe de assistência prénatal, devem registrar, no prontuário do paciente ou em outros documentos próprios, as informações inerentes ao processo de cuidar e ao gerenciamento dos processos de trabalho, e, assim, assegurar a continuidade e qualidade da assistência. ${ }^{24}$

A demanda de melhora na qualidade dos registros dos cartões de pré-natal, como ficou evidente neste estudo, pode ser obtida pela educação permanente em saúde. Essa educação deve ser pautada em metodologia ativa de ensino-aprendizagem, que valorize as experiências dos profissionais, e eleja os problemas do cotidiano na formulação de mudanças que impactem, nos modos de fazer, o complexo trabalho vivo em saúde. ${ }^{21,25} \mathrm{~A}$ construção desse conhecimento, na perspectiva freiriana, é também um ato político, pois considera o conhecimento como processo social, que implica na ação-reflexão do ser humano sobre o mundo, para transformar a realidade vivida, a partir do reconhecimento das contradições. ${ }^{26}$ Tais contradições foram evidenciadas, no caso deste estudo, na valorização de alguns registros em detrimento de outros igualmente importantes. Dessa forma, a abordagem pedagógica da situação deve envolver o processo de trabalho, a autonomia dos profissionais, os produtos gerados e os sentidos a eles conferidos, à luz das evidências científicas, para não causar danos decorrentes das diferenças no trabalho vivo.

Este estudo apresenta algumas limitações - tais como a impossibilidade de avaliar todos os cartões -, por motivos diversos, incluindo a ilegibilidade dos registros e a qualidade das fotografias, dificultando a leitura e interpretação dos registros. No entanto, a amostra estudada apresentou tamanho relevante, com iguais características sociodemográficas e assistenciais do total amostral do inquérito. Destaca-se, ainda, que esses achados foram similares aos poucos estudos que apresentaram similar proposta de avaliação. De qualquer forma, os resultados devem ser interpretados considerando que algumas mulheres, cujos cartões foram avaliados procediam de outros municípios da região metropolitana, limitando uma possível representação do pré-natal realizado em Belo Horizonte, mas sim dos nascimentos ocorridos no referido município.

\section{CONCLUSÃO}

Verificou-se a existência de grandes lacunas no registro do processo de assistência pré-natal nos cartões ou cadernetas do público avaliado, tendo em vista que procedimentos e parâmetros básicos das consultas de pré-natal deixam de ser registrados, gerando incerteza de que foram realizados. A omissão de registro de várias informações essenciais compromete a qualidade da assistência e o acompanhamento adequado das gestantes. Para averiguar a realização dos procedimentos não registrados, recomendam-se novos estudos que comparem os dados registrados na caderneta com as informações das mulheres.

Assim, os achados deste estudo apontam para a necessidade da educação permanente em serviço, direcionada aos profissionais de saúde - principalmente médicos e enfermeiros - quanto à importância da realização e registro das ações preconizadas na atenção pré-natal. $\mathrm{O}$ adequado e legível preenchimento das cadernetas de pré-natal aumenta a confiabilidade desse importante instrumento de comunicação entre profissionais e equipes na atenção obstétrica e perinatal, nos diferentes níveis de atenção à saúde. Por fim, talvez a base de todos os resultados esteja nos processos de trabalho ainda vigente de atenção à saúde, o qual prioriza as tecnologias duras em detrimento das tecnologias leves, sendo o registro uma tecnologia mais leve em relação à própria intervenção. 


\section{FINANCIAMENTO}

Conselho Nacional de Desenvolvimento Científico e Tecnológico (CNPq). Chamada Pública, Edital n.ํ20/2010, Processo $n$. - 402369/2010-6.

\section{AGRADECIMENTOS}

À Professora Dr. ${ }^{\text {a }}$ Edna Maria Rezende, que coordenou a pesquisa Nascer em Belo Horizonte, e permitiu a continuidade das pesquisas ao transferir sua coordenação após a aposentadoria.

\section{CONTRIBUIÇÕES DOS AUTORES}

Desenho do estudo. Letícia Ferreira de Camargos. Patrícia Lage Lemos. Eunice Francisca Martins. Mariana Santos FelisbinoMendes. Coleta de dados. Letícia Ferreira de Camargos. Patrícia Lage Lemos. Eunice Francisca Martins. Análise de dados e interpretação dos resultados. Letícia Ferreira de Camargos. Patrícia Lage Lemos. Mariana Santos Felisbino-Mendes. Redação e revisão crítica do manuscrito. Aprovação da versão final do artigo. Letícia Ferreira de Camargos. Patrícia Lage Lemos. Eunice Francisca Martins. Mariana Santos Felisbino-Mendes. Responsabilidade por todos os aspectos do conteúdo e a integridade do artigo publicado. Letícia Ferreira de Camargos. Patrícia Lage Lemos. Eunice Francisca Martins. Mariana Santos Felisbino-Mendes.

\section{EDITOR ASSOCIADO}

Aline Aparecida Monroe

\section{REFERÊNCIAS}

1. World Health Organization. WHO recommendations on antenatal care for a positive pregnancy experience [Internet]. Geneva: WHO; 2016 [citado 2020 maio 25]. Disponível em: https://apps.who.int/iris/bitstream/ handle/10665/250796/9789241549912-eng.pdf?sequence=1

2. Gonçalves LA, Costa LC, Ferreira VL, Abreu MS. Avaliação dos registros do número de consultas de pré-natal no cartão da gestante, sanitas e sisprenatal. Rev Admin Hosp e Inov em Saúde RAHIS. 2014 mar;11(4):266-77. http://dx.doi.org/10.21450/rahis.v11i4.2170.

3. World Health Organization. WHO recommendations on home-based records for maternal, newborn and child health [Internet]. Geneva:WHO; 2018 [citado 2020 maio 25]. Disponivel em: https://apps.who.int/iris/ bitstream/handle/10665/274277/9789241550352-eng.pdf?ua=1

4. Gonzalez TN, Cesar JA. Posse e preenchimento da Caderneta da Gestante em quatro inquéritos de base populacional. Rev Bras Saúde Mater Infant. 2019 jun;19(2):375-82. http://dx.doi.org/10.1590/180693042019000200007.

5. Santos No ET, Oliveira AE, Zandonade E, Gama SGN, Leal MC. O que os cartões de pré-natal das gestantes revelam sobre a assistência nos serviços do SUS da Região Metropolitana da Grande Vitória, Espírito Santo, Brasil? Cad Saude Publica. 2012 set;28(9):1650-62. http:// dx.doi.org/10.1590/S0102-311X2012000900005. PMid:23033181.

6. Ministério da Saúde (BR). Secretaria de Atenção à Saúde. Departamento de Atenção Básica. Atenção ao pré-natal de baixo risco. Brasília: Editora do Ministério da Saúde; 2012 [citado 2020 maio 25]. 318 p. Disponível em: http://bvsms.saude.gov.br/bvs/publicacoes/cadernos_atencao_ basica_32_prenatal.pdf

7. Valente MMPQ, Freitas NQF, Áfio ACE, Sousa CS, Evangelista DR, Moura ERF. Assistência Pré-Natal: um olhar sobre a qualidade. Rev
Rene Fortaleza. [Internet]. 2013; [citado 2020 maio 25];14(2):280-9. Disponivel em: http://periodicos.ufc.br/rene/article/view/3375/2613.

8. Andreucci CB, Cecatti JG. Desempenho de indicadores de processo do Programa de Humanização do Pré-natal e Nascimento no Brasil: uma revisão sistemática. Cad Saude Publica. 2011;27(6):1053-64. http:// dx.doi.org/10.1590/S0102-311X2011000600003. PMid:21710003.

9. Ministério da Saúde (BR). Caderneta da Gestante [Internet]. $4^{a}$ ed. Brasília: Ministério da Saúde; 2016 [citado 2020 maio 25]. 48 p. Disponível em: http://portalarquivos2.saude.gov.br/images/pdf/2018/agosto/31/ Caderneta-da-Gestante-2018.pdf

10. Viellas EF, Domingues RMSM, Dias MAB, Gama SGN, Theme Filha MM, Costa JV et al. Assistência pré-natal no Brasil. Cad. Saude Publica. 2014;30(suppl 1):S85-100. http://dx.doi.org/10.1590/0102$311 \times 00126013$

11. Leal MC, Szwarcwald CL, Almeida PVB, Aquino EML, Barreto ML, Barros F, et al. Reproductive, maternal, neonatal and child health in the 30 years since the creation of the Unified Health System (SUS). Ciênc saúde coletiva. 2018;23(6):1915-28. https://doi.org/10.1590/141381232018236.03942018

12. Bahk J, Yun SC, Kim Y, Khang YH. Impact of unintended pregnancy on maternal mental health: a causal analysis using follow up data of the Panel Study on Korean Children (PSKC). BMC Pregnancy Childbirth. 2015;15(1):85. http://dx.doi.org/10.1186/s12884-015-0505-4 PMid:25881099.

13. Carrilho JM. Avaliação dos Dados do Cartão de Pré-natal e Registro da Rotina Prevista na Assistência Pré-natal de Baixo Risco em uma Coorte de Gestantes em Belo Horizonte [dissertação]. Belo Horizonte (MG): Faculdade de Medicina da Universidade Federal de Minas Gerais; 2014.

14. Coêlho TTG, Medeiros ACQ, Ribeiro WCS, Menêzes TB. Avaliação do Grau de Completude do Cartão da Gestante de Puérperas Atendidas em um Hospital Universitário. R. Bras. Ci. Saúde. 2015;19(2):117-22. http://dx.doi.org/10.4034/RBCS.2015.19.02.05.

15. Amorim LP, Senna MIB, Gomes VE, Amaral JHL, Vasconcelos M, Silva AG et al. Preenchimento da Caderneta de Saúde da Criança nos serviços de saúde em Belo Horizonte, Minas Gerais, Brasil. Epidemiol Serv Saude. 2018;27(1):e201701116. http://dx.doi.org/10.5123/S167949742018000100016. PMid:29451613.

16. Amorim T, Felisbino-Mendes MS, Schreck RSC, Ribeiro SP, Rezende EM, Martins EF. Born in Belo Horizonte: the trajectory of parturient women and their reproductive outcomes. Rev Esc Enferm USP. 2019;53:e03441. http://dx.doi.org/10.1590/s1980-220x2018000903441.PMid:31166530.

17. Coutinho T, Monteiro MFG, Sayd JD, Teixeira MTB, Coutinho CM, Coutinho LM. Monitoramento do processo de assistência pré-nata entre as usuárias do Sistema Único de Saúde em município do Sudeste brasileiro. Rev Bras Ginecol Obstet. 2010 nov;32(11):563-9. http:// dx.doi.org/10.1590/S0100-72032010001100008. PMid:21271168.

18. Folk DM. Hypertensive disorders of pregnancy: overview and current recommendations. J Midwifery Womens Health. 2018;63(3):289-300. PMid:29764001.

19. Vettore MV, Dias M, Domingues RMSM, Vettore MV, Leal MC. Cuidados pré-natais e avaliação do manejo da hipertensão arterial em gestantes do SUS no Município do Rio de Janeiro, Brasil. Cad. Saúde Pública [online], 2011 maio;27(5):1021-34. https://doi.org/10.1590/S0102311X2011000500019.

20. Townsend R, O'Brien P, Khalil A. Current best practice in the management of hypertensive disorders in pregnancy. Integr Blood Press Control. 2016 jul 27;9:79-94. http://dx.doi.org/10.2147//BPC.S77344. PMid:27555797.

21. Merhy EE. Em busca do tempo perdido: a micropolítica do trabalho vivo em saúde. In: Merhy EE, Onoko R, organizadores. Agir em saúde: um desafio para o público [Internet]. São Paulo: Hucitec; 2002. p. 71-113 [citado 2020 maio 25]. Disponível em: https://digitalrepository.unm. edu/lasm_pt/145

22. Zanchi M, Gonçalves CV, Cesar JÁ, Dumith SC. Concordância entre informações do Cartão da Gestante e do recordatório materno entre puérperas de uma cidade brasileira de médio porte. Cad Saude Publica. 2013;29(5):1019-28. http://dx.doi.org/10.1590/S0102-311X2013000500019. PMid:23703007. 
23. Nunes JT, Gomes KRO, Rodrigues MTP, Mascarenhas MDM. Qualidade da assistência pré-natal no Brasil: revisão de artigos publicados de 2005 a 2015. Cad Saude Colet. 2016;24(2):252-61. http://dx.doi. org/10.1590/1414-462X201600020171.

24. Resolução COFEN n ${ }^{\circ} .429 / 2012$ de 8 de junho de 2012 (BR). Dispõe sobre o registro das ações profissionais no prontuário do paciente, $e$ em outros documentos próprios da enfermagem. Diário Oficial da União [periódico na internet], Brasília (DF), 8 jun 2012; Seção 1 [citado 2020 maio 25]. Disponível em: http://www.coren-ro.org.br/resolucao-cofenno-4292012_2155.html
25. Gigante RL, Campos GWS. Política de formação e educação permanente em saúde no Brasil: bases legais e referências teóricas. Trab Educ Saúde. 2016 set/dez;14(3):747-63. http://dx.doi.org/10.1590/1981. 7746-sip00124.

26. Freire P. A alfabetização de adultos: crítica de sua visão ingênua compreensão de sua visão crítica. In: Freire P. Ação Cultural para a Liberdade: e outros escritos [Internet]. Rio de Janeiro: Paz e Terra; 2003 [citado 2020 maio 25]. Disponível em: http:// www.gestaoescolar.diaadia.pr.gov.br/arquivos/File/otp/livros/ acao_cultural_liberdade.pdf 